\title{
Reply to the letter: Lycopene improves sperm motility and morphology: a promising agent for treating male infertility
}

\author{
Elizabeth A. Williams ${ }^{1}$ - Allan Pacey ${ }^{1}$
}

Published online: 22 January 2020

๑) Springer-Verlag GmbH Germany, part of Springer Nature 2020

We thank Zhai and Tang for their interest in our article "A randomized placebo-controlled trial to investigate the effect of lactolycopene on semen quality in healthy males" [1]. We too recognise that the study has several limitations. There was a small, but significantly higher BMI in the lactolycopene group which is difficult to interpret in the absence of information on body composition. We acknowledge that it would be useful to have information on physical activity of the participants; however, we did not include this to minimise the burden on participants and also avoid any assessments that may have prompted a change in habitual behaviour. Future studies may wish to consider the inclusion of more comprehensive assessment of body composition and physical activity. We agree that mechanistic studies and more empirical studies are in order, and that this study should be repeated on sub-fertile men. We hope that the study will encourage further investigation of the impact of lycopene on semen quality and will provide useful information to other researchers.

Elizabeth Williams and Allan Pacey

\section{Reference}

1. Williams EA, Parker M, Robinson A, Pitt S, Pacey AA (2019) A randomized placebo-controlled trial to investigate the effect of lactolycopene on semen quality in healthy males. Eur J Nutr. https ://doi.org/10.1007/s00394-019-02091-5 (Epub ahead of print)

This is a reply to a letter to the Editor available here: https://doi. org/10.1007/s00394-019-02173-4.

The original publication can be read here: https://doi.org/10.1007/ s00394-019-02091-5.

\section{Elizabeth A. Williams}

e.a.williams@sheffield.ac.uk

1 Department of Oncology and Metabolism, Faculty of Medicine Dentistry and Health, Medical School, The University of Sheffield, Beech Hill Road, Sheffield S10 2RX, UK 